Mon. Not. R. Astron. Soc. 000, 田罒(1996) Printed 11 October $2018 \quad$ (MN LATEX style file v1.4)

\title{
The Shapes and Ages of Elliptical Galaxies
}

\author{
Roelof S. de Jong and Roger L. Davies \\ University of Durham, Department of Physics, South Road, Durham DH1 3LE, England
}

Accepted Received in original form

\begin{abstract}
In this paper we investigate the relation between the detailed isophotal shape of elliptical galaxies and the strength of the $\mathrm{H} \beta$ absorption in their spectra. We find that disky galaxies have higher $\mathrm{H} \beta$ indices. Stellar population synthesis models show that the $\mathrm{H} \beta$ line is a good age indicator, hence disky galaxies tend to have younger mean ages than boxy galaxies. We show that the observed trend can be brought about by a contaminating young population, which we associate with the disky component. This population need only account for a small fraction of the total mass, for example if a contaminating population of age of 2 Gyrs is superimposed on an old (12 Gyr) elliptical galaxy, then the observed trend can be explained if it contributes only $10 \%$ to the total mass. The size of this effect is consistent with the estimates of disk-to-total light ratios from surface photometry.
\end{abstract}

Key words: galaxies: elliptical and lenticular - galaxies: evolution - galaxies: stellar content - galaxies: structure

\section{INTRODUCTION}

Recently a controversy has arisen over the ages of elliptical galaxies. The conventional view is that elliptical galaxies are old and coeval systems, formed by an initial starburst about 15 Gyrs ago. New population syntheses (Worthey 1994) combined with new observations of linestrengths in a large sam' ple of elliptical galaxies (González 1993), have brought this view into question (Faber et al. 1995; Worthey et al. 1995 ; for review see Davies 1996). These studies show that over a small range in metallicity, luminous elliptical galaxies span a wide range of $\mathrm{H} \beta$ absorption strengths implying ages from greater than 12 Gyrs to as young as 3 Gyrs. Many elliptical galaxies have isophote shapes that deviate from pure ellipses at the $1 \%$ level in the sense of being either "disky" or "boxy" (Bender et al. 1989). Here we investigate the hypothesis that the apparent young ages are brought about by the superposition of a young population (associated with the disky component) on an old elliptical population.

Determinations of the ages and metallicities of elliptical galaxies suffer from a degeneracy, both increased age and increased metallicity tend to redden the spectral energy distribution of a single stellar population and strengthen the metal absorption lines (Renzini 1986, Buzzoni et al. 1992, Worthey 1994). Recently, spectral indices have been identified that break this degeneracy. The stellar population synthesis models of Worthey 1994 indicate the $\mathrm{H} \beta$ and $[\mathrm{MgFe}]$ indices can be used in combination to determine age and metallicity separately. González (1993) collected longslit spectra of major and minor axis of a sample of about 40 elliptical galaxies. His observations indicate a substantial age spread among elliptical galaxies, as the $\mathrm{H} \beta$ index shows a large range of values over a small range in metallicity. This conclusion is difficult to reconcile with the hypothesis that elliptical galaxies have a single epoch of formation and are old, as has been inferred from the small scatter in their colour-magnitude, Fundamental Plane and $M g_{2}, \sigma$ relations (Bower et al. 1992a, 1992b; Renzini 1995). However in cosmological simulations where galaxies form through a hierarchy of mergers (Cole et al 1994; Kauffmann 1995; Baugh, Cole \& Frenk 1996), the stars that currently dominate the luminosity of giant galaxies could have a range of ages (if we associate star formation with those mergers that are gaseous). In such models, luminous elliptical galaxies form late (at redshifts $<1$ ) suggesting that at least the youngest populations, formed in the most recent merger, would be $<5$ Gyrs old. If elliptical galaxies consist of populations of different ages, the linestrengths will only reflect the luminosity weighted average age, not the age of formation or even the age of the last starburst. As young populations tend to be much more luminous, a small young population can dramatically change the strength of the lines (for detailed modeling see Charlot \& Silk 1994; Bressan, Chiosi \& Tantalo 1996. If there are several stellar population components in a galaxy one has to remain alert to the possibility that the $\mathrm{H} \beta$ and $[\mathrm{MgFe}]$ lines might not arise from the same stellar populations.

Elliptical galaxies have almost perfectly elliptical isophotes however small deviations from ellipses have been measured repeatably (Peletier et al. 1990, Goudfrooij et al. 1994). Higher order Fourier terms have been used to

(C) 1996 RAS 
parametrise these deviations. Unless isophotes are dramatically perturbed by the presence of dust, the higher order terms are small, only the fourth order cosine $(\cos (4 \theta))$ term showing significant deviations from zero. The maximum deviation in the $\cos (4 \theta)$ term as function of radius is taken as the characteristic value for the system, denoted by C4. Galaxies with disky isophote distortions have positive C4 values and those with boxy isophote distortions have negative values of $\mathrm{C} 4$.

Projection effects make the general interpretation of the C4 parameter non-trivial (Kochanek \& Rybicki 1996; Gerhard \& Binney 1996). The C4 interpretation of highly inclined systems is less ambiguous, positive C4's clearly result from disks embedded in the galaxy body. Even so, the $\mathrm{C} 4$ values cannot be translated directly into a disk-to-bulge (D/B) ratio. C4 only relates to the maximum deviation from ellipticity at a certain radius and the relation between $\mathrm{C} 4$ and $\mathrm{D} / \mathrm{B}$ ratio depends critically on the relative surface brightness and scalelengths of bulge and disk. We will use C4 values only as an indicator of disk presence, not as an direct indicator for $\mathrm{B} / \mathrm{D}$ ratios.

Here we explore how the diagnostic of youth, $\mathrm{H} \beta$ strength, is correlated with the deviation of the isophotes from perfect ellipses. In section two we present the correlation and in section three we explore how such a trend can be accounted for using Worthey's population models. In section 4 we consider the wider implications.

\section{$2 \quad$ H $\beta$ INDEX VERSUS ISOPHOTAL SHAPE}

To investigate whether there is a relation between isophotal shape and the $\mathrm{H} \beta$ index for elliptical galaxies, a large number of measurements were collected from the literature.

The C4 isophotal shape parameters were collected from Bender et al. (1989), Peletier et al. (1990) and Goudfrooij et al. (1994). Two different methods to evaluate C4 were used by these authors, and the difference produces a small scaling between the measured values. This was removed using a linear relation for the galaxies in common between the different authors (see also Peletier 1989). The maximum rms error between the measured C4 parameters of any two papers was 0.003 , which we will assume to be the typical uncertainty in the $\mathrm{C} 4$ parameter. The average of all available C4 measurements $(<\mathrm{C} 4>$ ) was used as best estimate of the C4 parameter of a galaxy.

The measurements of the equivalent width of the $\mathrm{H} \beta$ absorption line were mainly obtained from González (1993), with a few additional galaxies from Davies, Sadler \& Peletier 1993. González simulated aperture spectroscopy of his galaxies using longslit spectra. Azimuthal integration was simulated by taking luminosity and radially weighted averages of the linestrengths measured along the major and minor axis. We applied a similar procedure for the Davies et al. data, calculating linestrengths within the $1 / 8$ and the $1 / 2$ effective radius $\left(R_{\mathrm{e}}\right)$ of the galaxies. González corrected the $\mathrm{H} \beta$ absorption linestrengths for infilling due to emission by a relation between the strength of the $\mathrm{H} \beta$ line emission and that of the the [OIII] line at $5007 \AA$, namely $\Delta \mathrm{H} \beta=$ 0.7 [OIII]. We have adopted these corrected values. The validity of this correction has been questioned by Carrasco et al. (1995) who compared the change in $\mathrm{H} \beta$ with that in

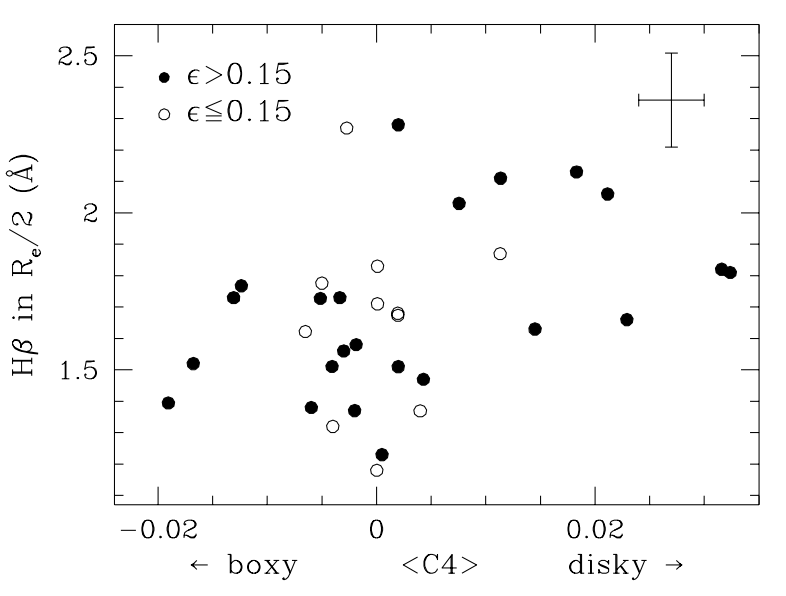

Figure 1. The average $\mathrm{C} 4$ index, determined as described in text, versus the $\mathrm{H} \beta$ equivalent width within $R_{\mathrm{e}} / 2$. The open symbols indicate galaxies with ellipticity $(\epsilon)$ less than 0.15 , solid symbols have ellipticity more than 0.15 , for which it is easier to determine C4. Typical error estimates are indicated in top-right corner.

$\mathrm{O}[\mathrm{III}]$ from one side of the nucleus to an equal radius on the opposite side in a sample of 26 galaxies. Assuming that the intrinsic $\mathrm{H} \beta$ absorption is symmetric about the nucleus they should have recovered the González relationship, however they did not, rather they found no correlation between the changes for galaxies with $[\mathrm{OIII}]<1.5 \AA$. The emission correction is rather uncertain as the range of $\mathrm{O}[\mathrm{III}] / \mathrm{H} \beta$ emission ratios in LINERS and HII regions is large (Baldwin, Phillips, Terlevich 1981; Ho, Filippenko \& Sargent 1993). We use the corrected values but note that the uncorrected (lower) values of $\mathrm{H} \beta$ result in age estimates that are considerably older than those discussed by González and collaborators and therefore establishing the correct $\mathrm{H} \beta$ emission correction is a fundamental step. In general the measurements of González and Davies et al. agree to within the stated errors, with a typical uncertainty of $0.18 \AA \mathrm{rms}$.

In Fig. 11 we plot the $<\mathrm{C} 4>$ parameter against the $\mathrm{H} \beta$ equivalent width within $R_{\mathrm{e}} / 2$ for all the galaxies for which we have data. For galaxies with ellipticities of $\epsilon \leqslant 0.15$ it is hard to determine C4, these are indicated by open circles. A trend is apparent in Fig. 1, for galaxies with $\epsilon>0.15$ the Spearman rank correlation coefficient is 0.48 indicating that the probability of finding such a correlation by chance is less than $2.5 \%$. All boxy galaxies have $\mathrm{H} \beta$ indices less than $1.8 \AA$, while the disky galaxies have often much higher indices, with no values below 1.6 $\mathrm{A}$. Similar trends are found when $R_{\mathrm{e}} / 8$ is used instead of $R_{\mathrm{e}} / 2$ or when the $\mathrm{H} \beta$ linestrengths are not corrected for emission.

\section{MODELS}

Can the $<\mathrm{C} 4>$ versus $\mathrm{H} \beta$ trend be understood in terms of simple stellar synthesis models by combining an old and a young population? If the high $\mathrm{H} \beta$ indices in disky galaxies are caused by a young disk component superposed on the old bulge component, we have to determine the typical mass and/or luminosity fraction of this disk within $R_{\mathrm{e}} / 2$ or $R_{\mathrm{e}} / 8$. A number of disk/bulge decomposition methods 
can be found in the literature (for review see Capaccioli \& Caon 1992). We will use the 2D bulge/disk decompositions of Simien \& Michard (1990) and of Scorza \& Bender (1995) to determine the typical range in Disk-to-Total (D/T) luminosity fraction of disky ellipticals in the region where the $\mathrm{H} \beta$ indices have been measured.

Simien \& Michard find D/T ratios of 0.14 for two elliptical galaxies. Scorza \& Bender find D/B ratios from 0.03 to 0.28 for their sample of disky ellipticals, which translates in $\mathrm{D} / \mathrm{T}$ ratios of about 0.03 to 0.22 . They were able to calculate local D/B ratios and concluded that these values were consistent with the values they derived from the independent analysis of line profile shapes determined from long slit spectra. They found typical local $\mathrm{D} / \mathrm{T}$ values within $R_{\mathrm{e}} / 2$ of 0.1 to 0.4 for disky ellipticals. We will use this range of values to form models of old and young populations using the models of Worthey (1994). Because younger populations are more luminous than older ones we use the $M / L$ ratios of the models to link the observed luminosity ratios to the mass ratios of the populations. Similarly we cannot simply take the average of the equivalent $\mathrm{H} \beta$ (or other) linewidths of the old and the young population to calculate the linestrengths of the combined population. Ideally we should add the spectra of the two populations scaled by the appropriate mass fractions, however as Worthey's models do not synthesise spectra but give magnitudes, colours and line indices, we have adopted a simpler approach. Before averaging two populations, we weight the linestrengths by the local continuum, which we approximate by using the $M / L_{V}$ ratios of the young and old populations. Starting with a 12 Gyr old population, we add a young population of between 0 and $20 \%$ in mass to calculate the resulting $\mathrm{D} / \mathrm{T}$ luminosity ratios and linestrengths.

Figure 1 shows the results of such calculations. Starting with a 12 Gyr old population of either solar or about 3 times solar metallicity, we add, in the top figure, solar metallicity populations of $1.5,2,3$ or $5 \mathrm{Gyr}$ and, in the bottom figure, 2 Gyr populations with $[\mathrm{Fe} / \mathrm{H}]=-0.225,0,0.25$ or 0.5 . The mass fraction of young-to-total population was varied from 0 to $20 \%$. The young populations have a strong effect on the luminosity fraction and the indices, even for small mass fractions. Different age and metallicity combinations for old and young populations illustrate the same basic result. A 3 Gyr old population can easily increase the $\mathrm{H} \beta$ index by $0.4-$ $0.8 \AA$, even with mass fractions of $20 \%$ or less. Making the old population even older than $12 \mathrm{Gyr}$ will shift the tracks down slightly so that a smaller young mass fraction is needed to produce the same effect in luminosity and $\mathrm{H} \beta . \mathrm{H} \beta$ is a good age indicator, with less sensitivity to metallicity than most other lines, but a lower metallicity young population still has considerably more effect on the $\mathrm{H} \beta$ equivalent width than a high metallicity young population.

Let us now compare the observed range in $\mathrm{H} \beta$ indices and $\mathrm{D} / \mathrm{T}$ ratios with these models. All boxy galaxies have $\mathrm{H} \beta$ indices less than $1.8 \AA$, consistent with the single burst 12 Gyr old populations of solar or super-solar metallicity. The disky galaxies have $\mathrm{H} \beta$ indices of $1.6-2.2 \AA$ within $R_{\mathrm{e}} / 2$ (and similar values within $R_{\mathrm{e}} / 8$ ), while the $\mathrm{D} / \mathrm{T}$ ratios are between 0.1 and 0.4 in these simulated apertures. These ranges have been indicated by the dotted lines in Fig. 2 . Clearly the full range in $\mathrm{H} \beta$ can be reached within the permitted range in $\mathrm{D} / \mathrm{T}$ light ratios. In the most extreme case of a (12 Gyr old plus a $1.5 \mathrm{Gyr}$ young) population, a $10 \%$
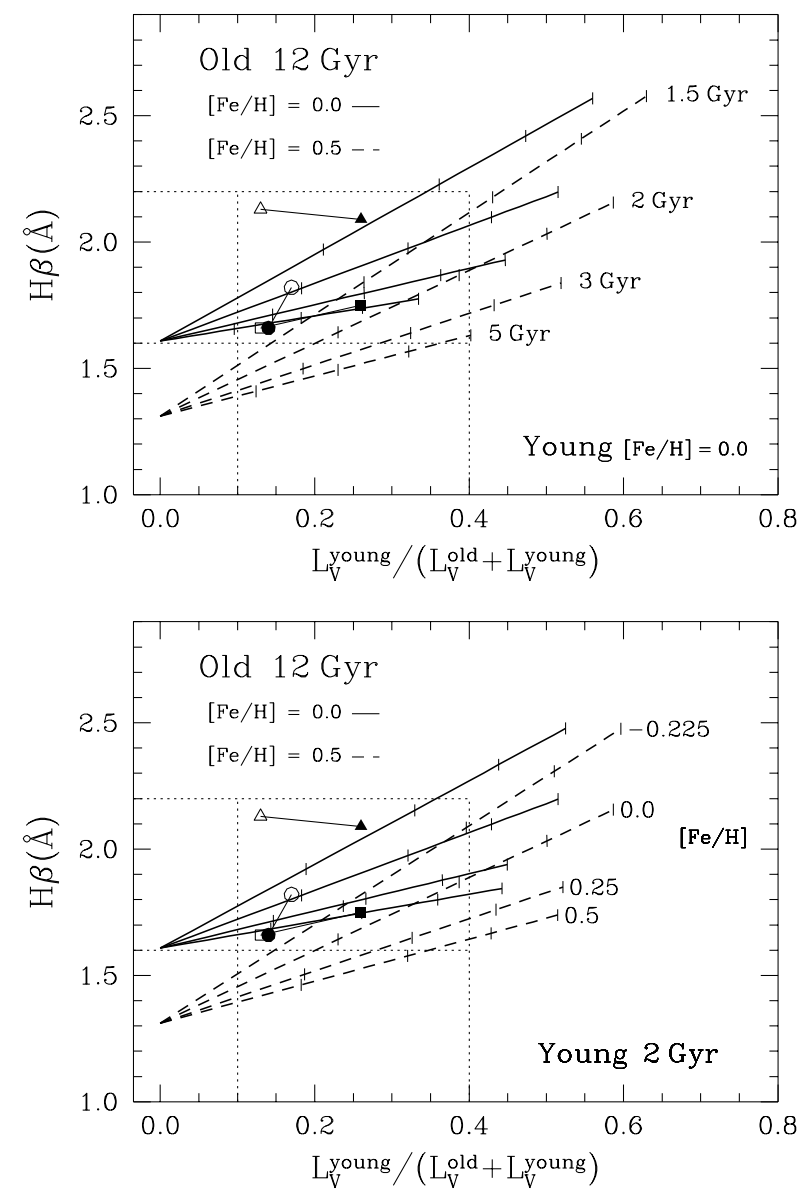

Figure 2. Model calculations of the combined effects of old and young populations, showing the young-to-total luminosity ratio versus $\mathrm{H} \beta$ equivalent width. All old populations are $12 \mathrm{Gyr}$, with either metallicity $[\mathrm{Fe} / \mathrm{H}]=0.0$ or 0.5 as indicated. In the top panel the young population is of solar metallicity, with ages $1.5,2,3$ or 5 Gyr from top to bottom. In the bottom panel all young populations have an age of $2 \mathrm{Gyr}$, but the metallicity varies with $[\mathrm{Fe} / \mathrm{H}]=-0.225,0.0,0.25$ and 0.5 from top to bottom. Each tickmark along the model lines corresponds to a $5 \%$ increase in the young-to-total mass of the stellar populations. The dotted lines indicate the observed range in $\mathrm{H} \beta$ linestrength and luminosity ratios in disky ellipticals. Data for three galaxies (NGC 821 circles, NGC 3377 triangles and NGC 4268 squares) are plotted, the solid symbols indicate the $R_{\mathrm{e}} / 8$ values, the open symbols the $R_{\mathrm{e}} / 2$ values (see text).

disk mass contamination is sufficient to cover the full $\mathrm{H} \beta$ range.

For three galaxies (NGC 821, NGC 3377 and NGC 4697) we have calculated the exact position in Fig. 2, using the D/B decompositions of Scorza \& Bender (1995). The procedure to calculate $\mathrm{D} / \mathrm{B}$ ratios within $R_{\mathrm{e}} / 2$ and $R_{\mathrm{e}} / 8$ was identical that used by González to calculate line indices in simulated apertures. D/B profiles along major and minor axis were extracted from the original D/B maps produced by Scorza \& Bender. These were luminosity and radially weighted to calculate average $\mathrm{D} / \mathrm{T}$ values within $R_{\mathrm{e}} / 2$ and $R_{\mathrm{e}} / 8$. NGC 821 and NGC 4697 lie in the region covered by the models, only the $R_{\mathrm{e}} / 2$ point of NGC 3377 can not be explained by the models. This galaxy has a large (and uncer- 


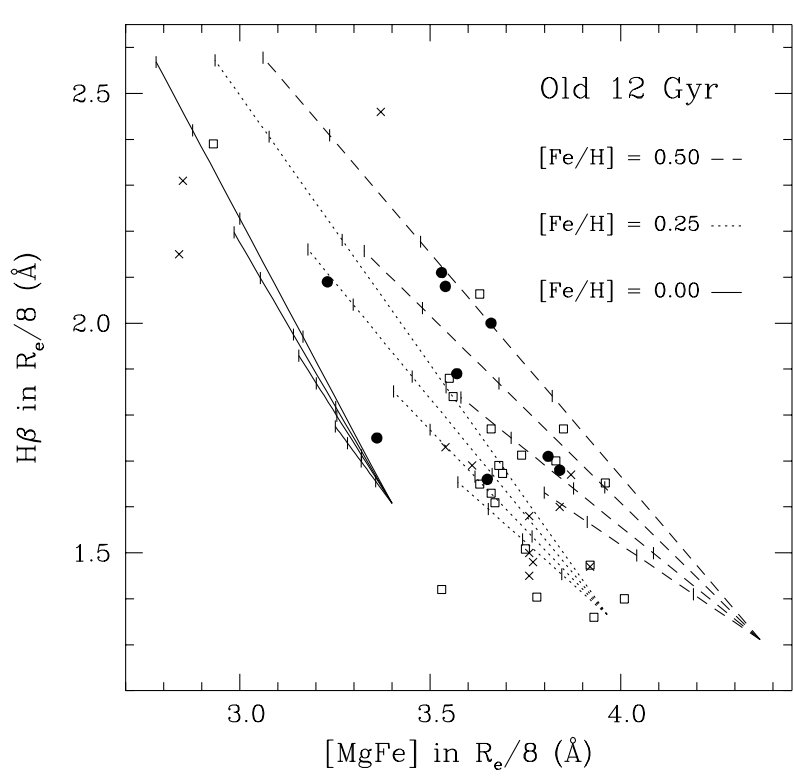

Figure 3. The $\mathrm{H} \beta$ versus $[\mathrm{MgFe}]$ equivalent width for all galaxies, solid circles the galaxies with $\langle\mathrm{C} 4\rangle \geqslant 0.005$, open squares $<\mathrm{C} 4><0.005$ and crosses galaxies with no measured C4. The lines show models, in which we combine $12 \mathrm{Gyr}$ old populations of the indicated metallicities with young populations of Solar metallicity. To each old population we have added, from top to bottom, a $1.5,2,3$, or 5 Gyr young population. Each tickmark along the model lines is $5 \%$ increase in the young-to-total mass of the combined populations.

tain) emission line correction of $0.35 \AA$, which might explain the discrepancy. A sub-solar metallicity in the main body of this galaxy would also remove the discrepancy.

We have shown that the modeled $\mathrm{H} \beta$ indices are consistent with the young disk interpretation and now explore consistency with the metal line indices. González (1993; see also Faber et al. 1995) uses a combination of the $\mathrm{Mg} b$ and the $\mathrm{Fe} 5270+\mathrm{Fe} 5335$ indices to formulate a $[\mathrm{MgFe}]$ index, empirically estimated to be a good metallicity indicator. Figure 3 shows the data for González galaxies with the above models superimposed. Consistent models for both a good age indicator $(\mathrm{H} \beta)$ and a metallicity indicator $([\mathrm{MgFe}])$ can be produced by combining an old metal-rich population with younger populations. Different combinations of age and metallicity and of old and young populations can cover the distribution of points without conflicting with the observed D/B decomposition results.

Due to the discreteness in age and metallicity of the populations calculated by Worthey (1994) we have opted here for simple combinations of young and old populations, however, continuous low level star formation or a series of small starbursts will produce similar effects in disk-to-total luminosity and integrated line indices. The point is that the old galaxy with young disk interpretation is consistent with the observations. One should be careful to interpret the line indices observations, which are heavily biased to the central regions of the galaxies, as indicative for the stellar population of the whole galaxy.

\section{DISCUSSION}

We have shown that the central $\mathrm{H} \beta$ indices of elliptical galaxies are correlated with their $\mathrm{C} 4$ parameters measuring isophotal shape. This suggests that the young ages found in some elliptical galaxies are associated with the disky components in these galaxies. Simple modelling, adding a small young population to an old population is capable of reproducing the change in the $\mathrm{H} \beta$ and metallicity indices by the required amount. These models are also consistent with the $\mathrm{D} / \mathrm{T}$ light ratios determined from the limited surface photometry available.

Given that the disky population of ellipticals is younger than the main body of the galaxy we are prompted to speculate on its origin. If it arises via mass lost from the host population that has settled into a disk by dissipation, we expect the gas to have high metallicity. However if the gas was accreted either from a low mass satellite galaxy or a reservoir of cold intergalactic gas we would expect a metallicity lower than that of the host. Disks formed from gas that has condensed out of cooling flows will also be metal poor, but will form over a extended period of time. Therefore if we were able to determine the metallicity of the disk (or gas) in a sample of such galaxies we would learn about the origin of these features. In either case not all elliptical galaxies are dormant star-piles. The possible range in disk age and metallicity is strongly dependent on the disk origin.

We conclude that further investigation of the ages of (disky) elliptical galaxies is required, using spatially resolved age indicators. Two possibilities are the comparison of the spatial distribution of line indices using long slit or integral field spectroscopy of galaxies and the colours of disks/bulges.

\section{ACKNOWLEDGMENTS}

We thank Cecilia Scorza for providing us the D/B ratio images of three galaxies. We thank Guy Worthey for making his models available to us.

\section{REFERENCES}

Baldwin, J.A., Phillips M.M., Terlevich R., 1981, PASP, 93, 5 Bender R., Surma P., Döbereiner S., Möllenhoff C., Madejsky R. 1989, A\&A, 217, 35

Baugh C.M., Cole S., Frenk C.S., 1996, MNRAS, in press

Bower R.G., Lucey J.R., Ellis R.S., 1992a, MNRAS, 254, 589

Bower R.G., Lucey J.R., Ellis R.S., 1992b, MNRAS, 254, 601

Bressan A., Chiosi C., Tantalo R., 1996, A\&A, in press

Buzzoni A., Gariboldi G., Mantegazza L., 1992, AJ, 103, 1814

Capaccioli M., Caon N., 1992, in Longo G., ed., 5th OAC Workshop on Morphological and Physical Classification of Galaxies. Reidel, Dordrecht, p. 99

Carrasco L., Buzzoni A., Salsa M., Recillas-Cruz E., 1995, in Buzzoni A., Renzini A., Serrano A., eds., Fresh Views of Elliptical Galaxies. ASP Conf. Series 86, p. 235

Charlot S., Silk J., 1994, ApJ, 432, 453

Cole S., Aragón-Salamanca A., Frenk C.S., Navarro J.F., Zepf S.E. 1994, MNRAS, 271, 781

Davies R.L., Sadler E.M., Peletier R.F., 1993, MNRAS, 262, 650

Davies R.L., 1996, in Davies R.L., Bender R., Proc. IAU Symp. 171, New light on galaxy evolution. Kluwer, Dordrecht, p. 37 
Faber S.M., Trager S.C., González J.J., Worthey G., 1995, in Gilmore G., van der Kruit P.C., eds., Proc. IAU Symp. 164, Stellar Populations. Kluwer, Dordrecht, p. 249

Gerhard O., Binney J.J., 1996, MNRAS, in press

González J.J., 1993, Ph.D. thesis, Univ. of California at Santa Cruz

Goudfrooij P., Hansen L., Jørgensen H.E., Nørgaard-Nielsen H.U., de Jong T., van den Hoek L.B., 1994, A\&AS 104, 179

Ho L.C., Filippenko A.V., Sargent W.L.W., 1993, ApJ, 417, 63

Kauffmann, G., 1995, MNRAS, 274, 161

Kochanek C.S., Rybicki G.R., 1996, preprint

Peletier R.F., 1989, Ph.D. Thesis, University of Groningen, The Netherlands

Peletier R.F., Davies R.L., Illingworth G.D., Davis L.E., Cawson M. 1990, AJ, 100, 1091

Renzini A., 1986, in Norman C., Renzini A., Tosi M., eds., Stellar Populations. Cambridge University Press, Cambridge, p. 213

Renzini A., 1995, in Gilmore G., van der Kruit P.C., eds., Proc. IAU Symp. 164, Stellar Populations. Kluwer, Dordrecht, p. 325

Scorza C., Bender R., 1995, A\&A, 293, 20

Simien F., Michard R., 1990, A\&A, 227, 11

Worthey G., 1994, ApJS, 95, 107

Worthey G., Trager S.C., Faber S.M., 1995, in Buzzoni A., Renzini A., Serrano A., eds., Fresh Views of Elliptical Galaxies. ASP Conf. Series 86, p. 203

This paper has been produced using the Royal Astronomical Society/Blackwell Science LTEX style file. 